\title{
The development of the relation between letter-naming speed and reading ability
}

\author{
KEITH E. STANOVICH \\ Oakland University, Rochester, Michigan 48063 \\ and \\ DOROTHY J. FEEMAN and ANNE E. CUNNINGHAM \\ University of Michigan, Ann Arbor, Michigan 48104
}

\begin{abstract}
A discrete-trial reaction time methodology was employed in order to measure the speed with which two groups of first-grade children (one tested twice during the school year) named letters. The relation of letter-naming speed to reading ability, although statistically significant, was much smaller than that observed in previous research in which a continuous-list procedure was employed. It was suggested that this procedure inflates the correlation because it involves many other psychological processes in addition to name retrieval speed. A third-grade group also displayed a weak correlation, but the relationship was reasonably strong in a fifth-grade group. The presence of a strong relationship in groups as advanced in reading as fifth-graders is probably more indicative of differential experience with text than it is of a causal role for name retrieval speed in determining reading ability.
\end{abstract}

Recently, there has been a good deal of research on the question of whether the speed of accessing the name code of a symbolic stimulus is related to reading ability. Although increasing in size, the literature on this issue remains confusing because the designs of the relevant studies differ on several different dimensions, making it difficult to integrate the results of the different experiments. For example, the developmental level of the subjects upon which individual research efforts have focused has varied widely, from adults (Jackson, 1980), to third-grade children (Perfetti, Finger, \& Hogaboam, 1978), to first-grade children (Speer \& Lamb, 1976; Stanovich, 1981). Some studies have concentrated on examining extremely disabled readers (e.g, Denckla \& Rudel, 1976), whereas other studies have formed ability groups based on the partitioning of the natural variation occurring in regular classrooms (e.g., Perfetti et al., 1978; Stanovich, 1981). In addition to subject differences, studies have varied in the tasks chosen to assess name-code access speed. Many studies have used various types of same-different matching tasks (e.g., Jackson, 1980), whereas others have used variants of vocalization tasks (Denckla \& Rudel, 1976; Perfetti et al., 1978).

When one considers the combinatorial possibilities provided by the dimensions of developmental level,

The authors would like to thank Karla M. Drayton for assistance in data collection. The authors wish to express appreciation to Cecilia Wiar, principal, and the teachers and students of Clarkston Elementary School, Clarkston, Michigan. Requests for reprints should be sent to Keith E. Stanovich, Department of Psychology, Oakland University, Rochester, Michigan 48063. reader ability differences, and task type, it will not be surprising that, despite an increasing number of studies in the past few years, few cells of the multidimensional matrix of subject-task situations are filled with enough data to yield unequivocal conclusions. It will be equally unsurprising that the overall structure of the matrix is unclear, given that so few cells are unambiguously anchored in data.

Given the current state of the literature, we decided to demarcate the present study in such a way as to provide detailed data on a subject-task situation of particular importance. Although we included third- and fifth-grade subjects in order to make a developmental comparison, the primary focus of the investigation was the relationship between name-code access speed and reading ability in first-grade children. Two groups of first-grade children were tested; one (the longitudinal group) was tested in the fall and late spring of the school year, and the other was tested once, in May. There were several reasons for concentrating on elucidating the nature of the relationship in the first grade. This period is one of obvious interest due to the importance of the early acquisition phase of reading. Second, despite the importance of first grade as a period of reading acquisition, remarkably few data exist on relationships involving name retrieval speed at this age.

It is additionally problematic that the few findings that do exist are contradictory. Biemiller (Note 1) found a correlation of -.56 between letter-naming time and scores on the reading comprehension subtest of the Metropolitan Achievement Test. Speer and Lamb (1976) found an even higher correlation (.86) between 
letter-naming speed and the comprehension section of the Gates-MacGinite Reading Test. However, Stanovich (1981) found a nonsignificant correlation (.17) between letter-naming time and the reading subtest of the Stanford Achievement Test. In a replication study that was a longitudinal investigation (Stanovich, Cunningham, \& West, 1981), letter-naming time measured in December displayed nonsignificant correlations with the reading subtest of the Stanford Achievement Test (-.21) and the Reading Survey of the Metropolitan Achievement Test (-.11). When letter-naming time was measured in March, these two correlations were -.04 and -.02 , respectively. In addition to the lack of convergence, the previous research is also disappointing in that fairly small sample sizes have been employed, 12 in the Biemiller (Note 1) study, 25 in the Speer and Lamb (1976) study, 22 in the Stanovich (1981) study, and 24 in the Stanovich et al. (1981) study. In the present study, both groups of first-grade subjects were twice as large as those employed in previous research.

A vocalization task was used to assess the speed of access to a name code. This would be the task of choice for reasons in addition to the fact that it insures comparability with previous results. Compared to other tasks, vocalization appears to be a relatively more "clean" and accurate index of the speed of access to a name code. The task appears to be less complex and less likely to be contaminated by other processes that might differentiate good from poor readers than are other tasks that have been employed. For example, same-different matching tasks have often been used to assess name-code access. However, such tasks involve any number of complex memory comparison and response decision processes that could differentiate good from poor readers apart from any differences in name retrieval speed that may be present. The main objection along these lines to vocalization tasks is that they may reflect reader-skill differences in vocal production latency in addition to name retrieval time. This criticism has been considerably obviated by several recent demonstrations that good and poor readers do not differ in articulation onset speed (Ellis, 1981; Mason, 1978). It thus appears that vocalization tasks may provide the most interpretable measures of name access speed. This is particularly advantageous in regard to studies of very young children, since the response requirements of vocalization tasks are simple and more natural than those of any other task.

Given that vocalization is to be the measure of name access speed, there still remain methodological choices to be made. Two general procedures have been used in vocalization experiments. One is a continuous-list procedure, in which the subject names a series of items displayed in rows and the total time to name the entire list is recorded (or the number of items named in a fixed time period). The other is the discrete-trial procedure, in which the reaction time to a single stimulus is recorded. It has previously been argued (Stanovich, 1981; Stanovich et al., 1981) that the discrete-trial procedure provides a much cleaner measure of name access time than does the continuous-list procedure. The continuous-list procedure involves complex scanning, sequential response, and motor-production strategies that could differentiate good from poor readers. Other investigators (e.g., Singer, 1982; Staller \& Sekuler, 1975) have pointed to several other processes that may become involved in a continuouslist task, thus serving to undermine the use of the task to isolate reading ability differences in the specific process of name access. Thus, the present study will employ a discrete-trial procedure, which eliminates some of the above-mentioned problems, but which necessarily involves name retrieval. ${ }^{1}$

The differences between the two types of vocalization tasks might not seem so critical had they yielded similar results. However, the tasks have not always produced similar data patterns. In fact, the discrepancies between the experiments on first-grade children may actually have their explanation in the different variants of the vocalization paradigm that have been used. Speer and Lamb (1976) and Biemiller (Note 1), who found strong correlations beween letter-naming speed and reading ability, both employed a continuous-list procedure, whereas in the Stanovich (1981) and Stanovich et al. (1981) studies, a discrete-trial procedure was used.

\section{METHOD}

Two groups of first-grade children served as subjects. They were from the same school but were tested during different years. One group (Group A) was tested twice during the school year, during late October/early November and during late May/ early June. There were 56 subjects ( 32 males and 24 females) in this group. They were recruited from three classrooms in a predominantly middle-class elementary school. The mean age of the children in May was 6 years 8 months. In May, all children were administered the comprehension subtest (Form 2, Primary Level A) of the Gates-MaoGinite Reading Tests and the Reading Survey (Form JS, Primary 1) of the Metropolitan Achievement Test. A median split of the group based on the Metropolitan Reading Survey scores yielded two groups, termed the skilled readers and less-skilled readers, respectively. The mean raw score of the skilled readers $(51.2$, grade equivalent $=3.4)$ differed significantly from that of the less skilled readers $(31.1$, grade equivalent $=1.8)[t(54)=13.93, p<.001]$. Similarly, the difference between the mean Gates comprehension score of the skilled readers $(34.0$, grade equivalent $=2.9)$ and the mean of the less skilled readers $(21.3$, grade equivalent $=1.7)$ was highly significant $[t(54)=10.91, p<.001]$.

The second group (B) of 53 first-grade children ( 28 males and 25 females) were tested during May and had a mean age of 7 years 4 months at that time. These children were all administered the Reading Survey (Form JS, Primary 1) of the Metropolitan Achievement Test in May. A split of the group based on this measure revealed that the mean raw score of the 27 skilled readers $(51.5$, grade equivalent $=3.9)$ was significantly different from that of the 26 less skilled readers $(30.2$, grade equivalent $=$ 1.8) $[\mathrm{t}(51)=14.27, \mathrm{p}<.001]$.

A group of 18 third-grade children (10 males and 8 females; mean age $=9$ years 1 month) were also tested in May and were administered the Reading Survey (Form JS, Elementary) of the Metropolitan Achievement Test. A median split of this group revealed that the mean raw score of the skilled readers $(49.4$, grade equivalent $=5.7$ ) was significantly different from that of the less skilled readers $(37.6$, grade equivalent $=3.2)[t(16)=$ $4.67, \mathrm{p}<.001]$. Twenty fifth-grade children $(10$ males and 
Table 1

Correlations Between Reading Ability and Letter-Naming Time for First-Grade Children

\begin{tabular}{llllll}
\hline \multicolumn{1}{c}{ Subject Group } & Time of Testing & N & \multicolumn{2}{c}{ Correlation } & Reading Ability Measure \\
\hline Group A & October/November & 56 & $-.38 \dagger$ & $(-.37) \dagger$ & Metropolitan (Gates) \\
Stanovich et al. (1981) & December & 24 & -.02 & $(-.04)$ & Metropolitan (Stanford) \\
Stanovich et al. (1981) & March & 24 & -.02 & $(-.04)$ & Metropolitan (Stanford) \\
Group B & May & 53 & $-.29 *$ & & Metropolitan \\
Group A & May/June & 56 & $-.28^{*}$ & $(-.18)$ & Metropolitan (Gates) \\
Stanovich (1981) & June & 22 & .17 & & Stanford \\
\hline
\end{tabular}

${ }^{*} p<.05 . \quad t p<.01$.

10 females; mean age $=11$ years 3 months) were tested in May and administered the Reading Survey (Form JS, Intermediate) of the Metropolitan Achievement Test. A median split of this group revealed that the mean raw score of the skilled readers $(53.0$, grade equivalent $=9.9)$ was significantly different from that of the less skilled readers $(35.2$, grade equivalent $=5.1)$ $[\mathrm{t}(18)=6.06, \mathrm{p}<.001]$.

The stimuli for Group A, the third-grade children, and the fifth-grade children were all the lowercase letters of the alphabet excluding the letters $i$ and 1 (deemed too confusable in the particular font that was used). The first four trials (employing the letters $s, q, e$, and c) were considered practice trials, and the remaining 20 trials (consisting of a fixed random ordering of the remaining letters) composed the experimental trials. All of the stimuli were typed in News Gothic font, photographed, and mounted on 35-mm white-on-black slides. Stimulus onset was controlled by a Vincent Associates Uniblitz shutter that was positioned over the lens of a projector. When the experimenter pushed a control button, the shutter was electronically opened, and the projected image of the stimulus item appeared. Simultaneously, a Lafayette Instruments electronic clock (Model 54419-A, accurate to the millisecond) was started by the same push of the control button. When the subject verbally responded, a voice-activated relay stopped the clock and closed the shutter.

The stimuli for Group B were uppercase and were presented on a BMC CRT monitor (with a refresh cycle of $16.7 \mathrm{msec}$ ) under the control of an Apple II microcomputer.

\section{RESULTS}

Trials on which the subject incorrectly named the stimulus, trials on which the response time was greater than $3,000 \mathrm{msec}$, and trials on which the response time was more than 3 standard deviations above the mean for that subject were scored as subject errors and dropped from the analysis. The performance of the third- and fifth-grade subjects was virtually errorless. The mean number of errors for the first-grade subjects in Group A was 3.1 in October/November and .84 in May/June. The mean number of errors for Group B was .72. The split-half reliabilities (Spearman-Brown corrected) of the reaction times were $.80, .92, .86, .94$, and .83 for Group A (October/November), Group A (May/June), Group B, third-grade subjects, and fifth-grade subjects, respectively.

For Group A, the correlation between the letternaming times and scores on the Metropolitan Reading Survey was $-.38(\mathrm{p}<.01)$ during the first testing and $-.28(\mathrm{p}<.05)$ during the second testing. Similar correlations were obtained when the letter-naming times were correlated with scores on the comprehension subtest of the Gates-MacGinite. The correlations between letternaming time and scores on the Metropolitan were $-.29(\mathrm{p}<.05)$ for Group $\mathrm{B},-.22$ (n.s.) for the thirdgrade subjects, and $-.54(p<.01)$ for the fifth-grade subjects.

A detailed view of the magnitude of the correlation at various points during the first-grade year is provided in Table 1, in which the data from Groups A and B are combined with the results previously reported by Stanovich (1981) and Stanovich et al. (1981), who used similar subject groups and procedures. The trends are easily summarized. The data from Groups A and B displayed correlations larger than those previously obtained by Stanovich (1981) and Stanovich et al. (1981), but markedly below those obtained in previous research using the continuous-list procedure (Speer \& Lamb, 1976; Biemiller, Note 1).

A few subjects in Group A produced an appreciable number of errors during the first testing. The performance levels of these subjects could possibly be more reflective of the degree of letter knowledge than of the ability to rapidly retrieve the name of a known stimulus. Thus, the correlations were recalculated after eliminating all nine subjects who made four or more letter-naming errors. The new correlation between letter-naming time and Metropolitan scores was $-.25(\mathrm{p}<.05, \mathrm{r}=-.27$ with Gates scores). It is possible that this figure is a more accurate estimate of the relation between first-grade reading ability and the speed of letter-name retrieval when the names have been learned to a reasonable degree of accuracy. Note also that naming errors are rarely reported in continuous-list experiments and that they probably strongly influence the overall time in such experiments due to hesitations, corrections, and uncertainty on the part of the subject.

An indication of the actual magnitude of the time

Table 2

Mean Naming Time (in Milliseconds) as a Function of Reader Skill

\begin{tabular}{lrrc}
\hline \multicolumn{1}{c}{ Subject Group } & Less & $\begin{array}{c}\text { Differ- } \\
\text { ence }\end{array}$ \\
\hline Group A-October/November & 934 & 1074 & $140 \dagger$ \\
Stanovich et al. (1981)-December & 1054 & 1102 & 48 \\
Stanovich et al. (1981)-March & 1017 & 991 & -26 \\
Group B & 680 & 740 & $60^{*}$ \\
Group A-May/June & 822 & 909 & $87^{*}$ \\
Stanovich (1981) & 775 & 774 & -1 \\
Third Grade & 661 & 771 & $110^{*}$ \\
Fifth Grade & 577 & 624 & $47^{*}$ \\
\hline
\end{tabular}

${ }^{*} p<.05 . \quad t p<.01$. 
differences that are involved in the naming time/reading ability relationship is given in Table 2 . This table displays the mean times for the skilled and less skilled readers as defined by median splits of each group. Again, the data of Stanovich (1981) and Stanovich et al. (1981) are included for purposes of comparison.

\section{DISCUSSION}

The correlations between letter-naming time and reading ability in Groups A and B were larger than those observed by Stanovich (1981) and Stanovich et al. (1981) and, unlike those from the previous research, were statistically significant. The reason for the discrepancy may be in the different sample sizes that were employed. It is clear from Table 1 that higher correlations were obtained for the larger subject groups. Perhaps it is primarily the children at the extremes of reading ability that contribute to the naming speed/reading ability relationship, and larger samples will necessarily contain more of these children.

Although the results of the present investigation confirmed the existence of a statistically significant relationship between letter-naming speed and reading ability in the first grade, the correlations were considerably smaller than those obtained in previous investigations (Speer \& Lamb, 1976; Biemiller, Note 1). This finding supports our previous conjecture, that the relationship between rapid name retrieval ability and reading skill is overestimated by continuous-list tasks that tap many other cognitive processes in addition to name retrieval. When a task that more specifically isolates the latter process is employed, the correlation appears to fall in the range of .20-.30 rather than in the $.60-.80$ interval suggested by the work of Speer and Lamb (1976) and Biemiller (Note 1). The fact that Table 1 indicates a quite modest relationship between name retrieval speed and reading ability in the first grade should also caution against making strong causal inferences about the influence of name retrieval ability when higher correlations are obtained at more advanced stages of reading acquisition (such as the -.54 correlation we obtained in our fifth-grade sample, or the many demonstrations that a relationship is present for adult subjects). Since the relationship is not present in strong form at the earliest stage of reading acquisition, subsequent increases in the correlation could be the result of the differential exposure to symbolic material received by readers of differing skill.

Finally, it should be noted that any relationship between letter-naming speed and reading ability is most probably due to differences in the name retrieval process and is not the result of other processes tapped by the letter-naming task. This is because several studies have ruled out the two most plausible alternative hypotheses. It was previously mentioned that no reader-skill differences in vocal production latency have been found in several studies. The other alternative hypothesis, that less skilled readers have a deficit in visually analyzing letters (i.e., in processes prior to name retrieval), has been contradicted by data from several empirical studies (e.g., Ellis, 1981; Stanovich, 1982; Vellutino, 1979).

\section{REFERENCE NOTES}

1. Biemiller, A. J. Relationships between reading time for letters, words, simple text, and word identification skill: $A$ 1-year longitudinal study. University of Toronto, unpublished manuscript, 1981.

\section{REFERENCES}

Denckla, M. B., \& Rudel, R. G. Rapid “automatized" naming (R.A.N.): Dyslexia differentiated from other learning disabilities. Neuropsychologia, 1976, 14, 471-479.

Ellis, N. Visual and name coding in dyslexic children. Psychological Research, 1981, 43, 201-218.

JACKSON, M. Further evidence for a relationship between memory access and reading ability. Journal of Verbal Learning and Verbal Behavior, 1980, 19, 683-694.

Mason, M. From print to sound in mature readers as a function of reader ability and two forms of orthographic regularity. Memory \& Cognition, 1978, 6, 568-581.

Perfetti, C. A., Finger, E., \& Hogaboam, T. Sources of vocalization latency differences between skilled and less skilled young readers. Journal of Educational Psychology, 1978, 70, 730-739.

Singer, M. Insensitivity to ordered information and the failure to read. In M. Singer (Ed.), Competent reader, disabled reader: Research and application. Hillsdale, N.J: Erlbaum, 1982.

SpeEn, O. B., \& LAMB, G. S. First grade reading ability and fluency in naming verbal symbols. Reading Teacher, 1976, 29, 572-576.

Staller, J., \& Sekuler, R. Children read normal and reversed letters: A simple test of reading skill. Quarterly Journal of Experimental Psychology, 1975, 27, 539-550.

Stanovich, K. E. Relationships between word decoding speed, general name-retrieval ability, and reading progress in firstgrade children. Journal of Educational Psychology, 1981, 73, 809-815.

Stanovich, K. E. Individual differences in the cognitive processes of reading I: Word decoding. Journal of Learning Disabilities, 1982, 15, 485-493.

Stanovich, K. E., Cunningham, A. E., \& West, R. F. A longitudinal study of the development of automatic recognition skills in first graders. Journal of Reading Behavior, 1981, 13, 57-74.

Vellutino, F. R. Dyslexia: Theory and research. Cambridge, Mass: M.I.T. Press, 1979.

\section{NOTE}

1. Note that we are not arguing that a discrete-trial vocalization task is a "pure" measure of name access time, but only that it is less contaminated with other processes and strategies than are other tasks and that differences in the relative degree of "contamination" may partially account for the apparent inconsistencies in the research literature (see below).

(Received for publication February 2, 1983.) 